\title{
Prenatal and postpartum maternal mental health and neonatal motor outcomes during the COVID-19 pandemic
}

\author{
Alissa Papadopoulos \\ Western University \\ Emily S. Nichols \\ Western University \\ Yalda Mohsenzadeh \\ Western University \\ Isabelle Giroux \\ University of Ottawa \\ Michelle F. Mottola \\ Western University \\ Ryan J. Lieshout \\ McMaster University \\ Emma G. Duerden ( $\nabla$ eduerden@uwo.ca ) \\ Western University
}

\section{Research Article}

Keywords: COVID-19, depression, infant, postpartum, pregnancy, infant, stress

Posted Date: February 17th, 2022

DOI: https://doi.org/10.21203/rs.3.rs-1351676/v1

License: (9) This work is licensed under a Creative Commons Attribution 4.0 International License.

Read Full License 


\section{Abstract \\ Background}

Rates of prenatal and postpartum stress and depression in pregnant individuals have increased during the COVID-19 pandemic. Perinatal maternal mental health has been linked to worse motor development in offspring, with motor deficits appearing in infancy and early childhood. We aimed to evaluate the relationship between prenatal and postpartum stress and depression and motor outcome in infants born during the COVID-19 pandemic.

\section{Methods}

One hundred and seventeen participants completed an online prospective survey study at two timepoints: during pregnancy and within 2 months postpartum. Depression was self-reported using the Edinburgh Perinatal/Postpartum Depression Scale (EPDS), and stress via the Perceived Stress Scale (PSS). Mothers reported total infant motor ability (fine and gross) using the interRAI 0-3 Developmental Domains questionnaire.

\section{Results}

Prenatal $(\mathrm{B}=-0.035,95 \% \mathrm{Cl}=-0.062$ to $-0.007, \mathrm{p}=.014)$ and postpartum maternal depression outcome $(B=-0.037,95 \% C l=-0.066$ to $-0.008, p=0.012)$ were significant negative predictors of total infant motor ability (fine and gross). Neither pregnancy nor postpartum perceived stress predicted infant motor function. A cluster analysis revealed that preterm and low birth weight infants belonging to mothers who reported elevated depressive symptoms during pregnancy and in the postpartum period had the poorest motor outcome.

\section{Conclusions}

Prenatal and postpartum depression, but not stress, predicted infant motor ability during the first 2 months of life. Preterm and low-birth weight infants of mothers who report elevated depressive symptoms are the most at-risk of experiencing poor motor outcome.

\section{Background}

Maternal mental health is an important predictor of infant and child development, as well as future health and educational outcomes (Burger et al., 2020; Ovetunji \& Chandra, 2020). Studies conducted over the past year suggest that the COVID-19 pandemic has had a tremendous adverse impact on the mental health of pregnant and postpartum individuals worldwide (Lebel et al., 2020; Stepowicz et al., 2020; Suárez-Rico et al., 2021; Wu et al., 2020; Zanardo et al., 2020; Zilver et al., 2020). Compared to before the 
pandemic, this work suggests that pregnant and postpartum individuals throughout the world are experiencing elevated levels of depression (Lebel et al., 2020; Wu et al., 2020; Zanardo et al., 2020). Stress levels have also increased, with many experiencing moderate to high levels of perceived stress (Stepowicz et al., 2020; Suárez-Rico et al., 2021; Zilver et al., 2021). Given the impact of perinatal stress and depression on offspring outcomes, the COVID-19 pandemic has significant potential to adversely affect the infants born as a result of a pregnancy affected by the COVID-19 pandemic.

Previous studies conducted during significant stressful events, such as natural disasters, maternal mental health and stress in particular was associated with worse motor functioning in infancy and childhood (Cao et al., 2014; Simcock et al., 2016). These deficits appear to begin early in infancy and can last into childhood (Cao et al., 2014; Simcock et al., 2016). Project Ice Storm which investigated prenatal maternal stress during the 1998 Quebec Ice Storm, showed that higher maternal perceived stress during pregnancy was associated with poorer motor outcome in 5.5-year-old children, particularly bilateral motor coordination (Cao et al., 2014). In the 2011 Queensland Flood Study, researchers reported a statistically significant association between maternal perceived stress and motor abilities at 2-, 6-, and 16-months of age (Simcock et al., 2016). However, the relationship between maternal perceived stress and motor abilities differed depending on age at assessment. At 2 months of age, higher maternal perceived stress was associated with better motor development, while at 6- and 16-months, stress was linked with poorer motor abilities (Simcock et al., 2016). Studies of maternal stress and motor outcome outside of disasters have reported similar results. In one study of 2-year-old children, researchers found that perceived workrelated stress at the time of delivery was negatively associated with motor ability (Chuang et al., 2011). Racine and colleagues (2018) found that fine motor abilities were poorer in 4-month-old infants whose mothers reported high levels of perceived stress and low social support during both pregnancy and the postpartum period.

Maternal depression is also an important predictor of offspring motor outcome during infancy and childhood (Abrams et al., 1995; Chuang et al., 2011; Cornish et al., 2005; Diego, Field \& Hernandez-Reif, 2005; Field et al., 2004; O'Leary et al., 2019; Pinheiro et al., 2014; Racine et al., 2018). Maternal depression during pregnancy is associated with poorer motor abilities in newborns (Abrams, 1995; Field et al., 2004) and at 6 months of age (O'Leary et al., 2019). Postpartum depression has also been linked to decreased motor abilities (Slomian et al., 2019) at 6-8 months (Nasreen, Kabir, Forsell \& Edhborg, 2013) and 12-18 month of age (Ali, Mahmud, Khan \& Ali, 2013; Cornish et al., 2005; Koutra et al., 2013). Researchers have suggested that the combination of depression during both pregnancy and the postpartum period put infants at even higher risk of decreased motor abilities relative to either period alone. Diego, Field \& Hernandez-Reif (2005) showed that at 2 weeks, infants born to mothers depressed both during and after pregnancy displayed worse motor abilities than infants of non-depressed mothers. The infants of mothers depressed during both pregnancy and postpartum periods also had lower scores than infants of mothers who were depressed only at the prenatal or postpartum period, however this difference was not statistically significant. 
In the current study we examined the association between prenatal and postpartum maternal stress and depression and neonatal motor outcome in the first two months after birth. This is the first study, to our knowledge, to assess neonatal outcome related to maternal mental health during the COVID-19 pandemic. Compared to other disasters that have been studied in relation to maternal mental health and infant development, including the 1998 Quebec Ice Storm and the 2011 Queensland Flood, the COVID-19 pandemic has been the longest enduring disaster, with no current or definitive end in sight. Gaining a further understanding in the ways that the COVID-19 pandemic is influencing mothers and infants can help us know when to intervene to help mitigate the long-term deleterious effects that the COVID-19 pandemic is having on mothers and infants alike. We addressed two main research questions: 1) Does prenatal maternal stress or depression predict neonatal motor outcome? 2) Does postpartum maternal stress or depression predict neonatal motor outcome? We hypothesized that prenatal and postpartum stress and depression would negatively predict neonatal motor functioning.

\section{Methods}

\section{Participants}

Participants in this online prospective survey study were pregnant persons with singleton or multiple pregnancies who were 18-55 years old. Individuals any stage of pregnancy were invited to participate. Participants had to be able to read and write in English and have an internet connection to participate, otherwise no other exclusionary criteria were applied. Participants were recruited through Prolific, Amazon Mechanical Turk, and through social media advertisements. Data were collected between May 2020 March 2021. All participants provided their written informed consent online. All procedures involving human subjects were approved by the Research Ethics Board at Western University (REB \#115810) and this study was conducted in accordance with the Helsinki Declaration of 1975, as revised in 2008.

\section{Procedures}

The online survey data was collected via Qualtrics (Qualtrics, Provo, UT) at two timepoints: the first was at any time during pregnancy, and the second was within 2 months of giving birth. At time 1, information about the participant's gender, age, ethnicity, city and country of residence, education, occupation, gestational age, pregnancy complications, physical health, mental health, and stress, was obtained. At time 2, maternal mental health and stress was assessed, and we obtained information on neonatal characteristics, such as birth weight and gestational age, and neonatal motor outcome.

\section{Demographic Measures}

The Barratt Simplified Measure of Social Status (BSMSS) was used as a measure of socioeconomic status (Barratt, 2006) at the first timepoint. Based on the BSMSS scoring system, participants were given a score between 8-66 based on their occupation and education level, with a higher score indicative of a higher SES.

\section{Maternal Depression and Stress and Infant Motor Function}


At both timepoints, maternal stress was assessed using the Perceived Stress Scale (PSS), a 10-item selfreport measure where participants rate each item on a scale of $0-4$ points (Cohen, Kamarck \& Mermelstein., 1983). The 10-item version of the PSS has demonstrated very good reliability and validity (Mitchell et al., 2008). When completing the questionnaire, participants are asked to consider their stress during the last month. The PSS consists of 6 negatively stated items (i.e., "how often have you found that you could not cope with all the things that you had to do?") and 4 positively stated items (i.e., "how often have you felt confident about your ability to handle your personal problems?"). The negatively stated items are scored as 0 = never, $1=$ almost never, $2=$ sometimes, $3=$ fairly often, and $4=$ very often, and scoring is reversed for the positively stated items. The total score for the PSS is the sum of the raw score from all 10 of the items. A higher score on the PSS is indicative of more perceived stress. Scores 13 and under are considered low perceived stress, scores 14-26 are considered moderate perceived stress, and scores 27 and above are considered high perceived stress.

Maternal depression was assessed using the Edinburgh Perinatal/Postpartum Depression Scale (EPDS) at both timepoints (Cox, Holden \& Sagovsky, 1987). The EPDS is a self-report questionnaire that consists of 10 items rated on a scale from 0-3 points. When filling out the EPDS, participants are asked to consider their symptoms of depression and their frequency over the past 7 days. The total score for the EPDS is the sum of the raw score from each of the 10 items. Total scores of 14 or higher are considered as probable clinical antenatal depression (Murray et al., 1990; Adewuya et al., 2006; Felice et al., 2006).

\section{Total Neonatal Motor Outcome}

To assess neonatal motor outcome, we used the Gross and Fine Motor Scales from the interRAI 0-3 Developmental Domains questionnaire (Stewart et al., 2017). Only the questions that corresponded to infants between 0-2 months of age were used as all infants in the study were between the ages of 0-2 months. The Gross Motor Scale for infants between 0-2 months consists of 4 questions that assess the startle reaction, trunk and extremity movement, side-to-side head movement, and head lifting. The Fine Motor Scale for infants between 0-2 months consists of 2 questions that asses resting hand position and palmer grasp. Participants were asked to consider their baby's highest level of performance and to rate each item as yes if they had observed their baby perform the movement within the last 3 days, and as no if they had not observed the behaviour in the last 3 days. Each item in the Gross Motor and Fine Motor Scales were designated a score of 0 or 1 . A score of 1 indicates that the parent observed this behaviour in their infant and a score of 0 indicates that the parent has not observed the behaviour. The Gross and Fine Motor Scales were combined to arrive at a Total Neonatal Motor Outcome score, that was calculated as a sum of the raw score from both the Gross and Fine Motor Scales. The highest achievable score was 6 , and a higher score was indicative of better motor abilities in the infant. The Gross and Fine Motor Scales were combined due to the small number of questions in each scale.

\section{Statistical Analysis}

Statistical analyses were completed the IBM SPSS Statistics software package (version 27, Statistical Package for the Social Sciences, IBM, Armonk, NY). 
Our first aim was to examine if pregnancy depression or stress predicted Total Neonatal Motor Outcome. In two general linear regression models, we examined Total Neonatal Motor Outcome (dependent variable) in relation to prenatal perceived stress and prenatal depression (independent variables) in separate models. All models were adjusted for maternal age, SES (using the BSMSS), country of residence, trimester of pregnancy, and month of survey completion according to our previous methods (Papadopoulos et al., 2021). The models were corrected for multiple comparisons.

Our second aim was to examine if postpartum mental health is associated with Total Neonatal Motor Outcome. Using linear regression models, we examined Total Neonatal Motor Outcome (dependent variable) in relation to postpartum perceived stress and postpartum depression (independent variables) in separate models. In both models maternal age, SES (using the BSMSS), country of residence, and month of survey completion, gestational age at birth (Papadopoulos et al., 2021). The models were corrected for multiple comparisons. Post hoc power calculation was performed using $G^{*}$ Power to determine the effect size.

\section{Results}

\section{Participant Characteristics}

A total of 1185 pregnant participants were enrolled in the study, and $869(73 \%)$ completed the full survey at time 1 during pregnancy. Partial datasets from both time points were available in $117(13 \%)$ participants. The majority of the 117 participants who completed time 1 (median age $=31.0$ years, interquartile range $[\mathrm{IQR}]=29.0-34.7$ years) and time 2 (median age $=31.0$ years, IQR $=29.0-35.0$ years) were in the third trimester of pregnancy $(n=79,67.5 \%)$ when they completed timepoint 1 . Participants resided in Canada, United States, United Kingdom, India, and other countries in Europe, South America, and Asia. Detailed participant characteristics from times 1 and 2 can be found in Table 1.

\section{Prenatal Stress}

With a Wilcoxon Signed Ranked Test, we determined that that both levels of perceived stress $(Z=-4.32, p<$ $0.001)$ and depression $(Z=-4.21, p<0.001)$ decreased in time 2 compared to timepoint 1 in our sample.

\section{Pregnancy Depression, Stress \& Neonatal Motor Function}

In our first aim, we examined whether prenatal perceived stress and prenatal depression could predict neonatal motor abilities at 0-2 months of age. A total of 104 participants were included in the analysis. We found that prenatal depression was a statistically significant negative predictor of Total Neonatal Motor Outcome $(B=-0.035,95 \% \mathrm{Cl}=-0.062$ to $-0.007, p=.014)$; however, prenatal perceived stress was not significantly associated with Total Neonatal Motor Outcomes $(B=-0.017,95 \% \mathrm{Cl}=-0.038$ to $0.004, p=$ 0.116).

\section{Postpartum Depression, Stress \& Total Neonatal Motor Outcome}


In our second aim, we examined whether postpartum perceived stress and postpartum depression could predict Total Neonatal Motor Outcome at 0-2 months of age. A total of 95 participants were included in the analysis. We found that postpartum depression was a significant negative predictor of neonatal motor outcome $(B=-0.037,95 \% \mathrm{Cl}=-0.066$ to $-0.008, p=0.012)$; however postpartum perceived stress was not associated with Total Neonatal Motor Outcome $(B=-0.007,95 \% \mathrm{Cl}=-0.029$ to $0.16, p=0.560)$.

\section{Post-Hoc Cluster Analysis}

As part of an exploratory analysis, a K-means cluster analysis, a data-driven approach, with 107 participants was conducted to identify the characteristics of participants who are most at risk of impairments in motor outcome. The analysis was run with Z-scored variables, including prenatal depression $(p<0.001)$, postpartum depression $(p<0.001)$, Total Neonatal Motor Outcome $(p<0.001)$, birth weight (kgs) $(p<0.001)$, and gestational age at birth $(p<0.001)$ and consisted of four clusters. Refer to Fig. 1 for a diagram of the clusters and to Table 2 for the demographic information of participants within each cluster.

Cluster 1 included 17 participants. Participants in this cluster were characterized by low-average levels of pre- and post-natal depression (i.e., Z-score <0)., average Total Neonatal Motor Outcome (i.e., Z-score at approximately 0 ), lower birth weight and lower gestational age at birth. Approximately $70.6 \%$ of the participants in cluster 1 had lower than average prenatal depression scores, and $82.4 \%$ had lower than average postpartum depression scores. Only 1 participant from this group had motor outcome scores below average. All but one participant in cluster 1 had a birthweight that was below average, and all participants were born earlier than the average gestational age at birth.

Cluster 2 included 49 participants. Participants in this cluster were characterized by very low levels of preand post-natal depression, average Total Neonatal Motor Outcome, average birth weights and average gestational ages at birth. $79.6 \%$ and $83.7 \%$ of the participants in Cluster 2 had prenatal and postpartum depression scores that were lower than the average, respectively. In this group, 42 participants had average motor outcome scores. While 7 participants in cluster 2 were below average on motor outcome scores they were all less than 1 standard deviation (SD) below average. The birth weights of participants in cluster 2 ranged from 2.2 SD above average to approximately 1 SD below average and gestational age and $69.4 \%$ had a gestational age that was at least average at birth.

Cluster 3 included 27 participants. Participants in this cluster were characterized by high levels of both pre- and post-natal depression (i.e., Z-score of more than 0 ), average motor outcome scores, average birth weight and average gestational age at birth. All but 3 participants had higher than average prenatal depression scores, with 33.3\% scoring at least 1 SD higher than the average. In addition, all but 2 participants had higher than average postpartum depression scores, with $59.3 \%$ scoring at least $1 \mathrm{SD}$ higher than average. Only 4 participants in this cluster had motor outcome scores lower than the average. $40.7 \%$ of participants in cluster 3 had birth weights that were above average, and $77.8 \%$ of participants in this cluster had average gestational ages at birth. 
Cluster 4 included 14 participants. Participants in this cluster were characterized by high levels of both pre- and post-natal depression scores, impaired motor outcome scores, low birth weight, and low-average gestational age at birth. Prenatal depression scores were higher than average in $71.4 \%$ of participants in cluster 4 , and postpartum depression scores were higher than average in $42.9 \%$ of the participants in this cluster. All participants in this cluster had motor outcome scores below the average, with 8 participants being at least $1.8 \mathrm{SD}$ below the average, and 4 of these participants being more than 3 SD below average. $71.4 \%$ of cluster 4 participants had lower than average birth weights, and $50 \%$ of the sample was born at an earlier gestational age than the average.

A one-way ANOVA revealed significant differences between the clusters on all the variables included in the analysis: EPDS prenatal score $(F(3,103)=29.09, p<0.001)$, EPDS postpartum score $(F(3,103)=27.84$, $p<0.001)$, motor outcome score $(F(3,103)=62.36, p<0.001)$, birth weight $(F(3,103)=15.46, p<0.001)$, and gestational age at birth $(F(3,013)=27.68, p<0.001)$. Post-hoc multiple comparisons using Bonferroni correction revealed significant differences between the clusters. Significant differences were found between Clusters 1 and 2 on birth weight and gestational age at birth (both $p<0.001$ ), whereby the infants in Cluster 1 had significantly lower birthweights and lower gestational ages at birth.

Clusters 1 and 3 significantly differed on EPDS prenatal depression scores, EPDS postpartum depression scores, birth weight, and gestational age at birth (all $p<0.001$ ), whereby the participants in Cluster 1 had lower EPDS scores (prenatal and postpartum), but their infants had lower birthweights and lower gestational ages.

Clusters 1 and 4 significantly differed on EPDS postpartum depression score $(p=0.034)$, motor outcome score $(p<0.001)$, and gestational age at birth $(p<0.001)$. Participants in Cluster 4 had higher postpartum depression scores and their infants had lower motor scores and were born significantly later than babies in Cluster 1.

Clusters 2 and 3 significantly differed on EPDS prenatal depression score and EPDS postpartum depression score (both $p<0.001$ ). Participants in Cluster 3 had significantly higher EPDS (pre and postpartum) scores compared to participants in Cluster 2.

Clusters 2 and 4 significantly differed on EPDS prenatal depression score and EPDS postpartum depression score, motor outcome score, birth weight (all $p<0.001)$, and gestational age at birth $(p=$ 0.037). Participants in Cluster 2 had lower EPDS (pre-, postnatal) scores and their infants had higher motor outcome scores, higher birthweights and older gestational ages compared to the participants in Cluster 4.

Clusters 3 and 4 only significantly differed on motor outcome score $(p<0.001)$, whereby infants in Cluster 4 had significantly lower motor scores.

\section{Discussion}


We conducted a prospective cohort study with pregnant women during the COVID-19 pandemic to better understand maternal distress during this period and the association with neonatal outcomes. In a subset of the cohort whereby longitudinal data were available, higher levels of maternal prenatal and postpartum depression were associated with worse infant motor outcomes within the first 2 months of life. However, maternal prenatal and postpartum perceived stress were not significant predictors of infant motor outcome in the 2 months after birth. Finally, we identified a group of infants that were at a higher risk of atypical motor development. These infants were born at an early gestational age, had low birth weights, and had mothers with elevated levels of depressive symptoms both prenatally and in the postpartum period.

\section{Prenatal and Postpartum Maternal Depression}

As hypothesized, both prenatal and postpartum maternal depression negatively predicted neonatal motor functioning. This is consistent with previous research which has demonstrated a negative correlation between both prenatal (Abrams et al., 1995; Field et al., 2004; O'Leary et al., 2019) and postpartum maternal depression (Ali, Mahmud, Khan \& Ali, 2013; Cornish et al., 2005; Diego, Field \& Hernandez-Reif, 2005; Koutra et al., 2013; Nasreen, Kabir, Forsell \& Edhborg, 2013) and infant and child motor outcome. These results add to the literature on the effects of maternal depression on early motor outcome in neonates. Future research should assess the motor outcome of these participants as they further age and develop.

Our cluster analysis revealed that the neonates most at-risk for adverse motor outcome were those who were born at earlier gestational ages with lower birth weights and whose mothers were depressed both prenatally and at postpartum, which was observed in cluster 4 . Neonates with low birth weight and who were born early but did not have mothers who were experiencing elevated depressive symptoms either prenatally or during postpartum, as in cluster 1, did not show impaired motor outcomes. Neonates in cluster 3 who were born to mothers with high depressive symptoms both prenatally and postpartum but were born at a normal birthweight and at term also did not show impairments on motor outcome. This suggests that the combination of birth factors (i.e., low birth weight and early delivery) and maternal depression might put neonates at an elevated risk for abnormal development and should be further examined.

Research suggests that depressed mothers are less responsive to their infants, which could result in an insecure attachment between infant and mother (Nasreen, Kabir, Forsell \& Edhborg, 2013; Sacchi et al., 2018). This insecure attachment could result in infants who are less comfortable and less encouraged to engage in motor-exploration. For the infants in cluster 3 , the combination of potential decreased motor exploration compounded by being physically smaller and weaker, due to low birth weight and early delivery, could have led to the results found in the present study. Research also suggests infant temperament interacts with symptoms of maternal depression to predict infant motor ability (Sacchi et al., 2018). These data were not available in the current study; however, this is an important area for follow-up research. 


\section{Prenatal and Postpartum Maternal Perceived Stress}

Research suggests that high levels of maternal perceived stress, which are often accompanied by elevated cortisol levels, can have a negative impact on infant and child motor development via atypical maturation of the cerebellum (Chuang et al., 2011; Cao et al., 2014; King et al., 2012; Racine et al., 2018; Simcock et al., 2016). In the present study, higher prenatal and postpartum perceived stress scores were negatively associated with Total Neonatal Motor Outcome, however, neither of these associations reached statistical significance.

The effects of maternal perceived stress on motor outcome may depend on the timing of the stress during pregnancy, with stress later in gestation leading to poorer motor ability (Cao et al., 2014). In our analyses, trimester was not a significant covariate in the model with motor outcome and prenatal maternal perceived stress. However, the timing of our assessment of perceived stress during gestation was not precise and the participants were not followed long-term to monitor changes throughout pregnancy. Therefore, it is possible that we did not capture high levels of perceived stress later in gestation that were related to motor outcome.

In addition, research suggests that prenatal maternal perceived stress differentially influences motor outcome depending on the age that the infant or child is assessed (Simcock et al., 2016; King et al., 2012). In a study by Simcock and colleagues that examined perceived stress and motor outcome in infants born during the Queensland flood, higher maternal perceived stress during pregnancy was related to better motor outcome at 2-months of age. However, by 6- and 16-months of age, the relationship changed, and stress was associated with poorer motor abilities. In our study the neonates were assessed from birth up to 2 months of age. It is possible that it was too early to detect the effects of stress on motor outcome at this point in development and is possible that the relationship between maternal perceived stress and motor outcome in these neonates may change as they age. More time might also be needed to examine the effects of postpartum perceived maternal stress on the motor abilities of these neonates as they further develop.

\section{Limitations}

There are a number of limitations that must be taken into account. First, we had a high rate of attrition. Of the 1185 participants enrolled in the study at time point 1, only 117 participants returned to complete time point 2 measures. Second, while the inteRAl provides both fine and gross motor scales, we combined these scales in order to provide an overall measure of motor ability in our neonates. Third, although the interRAI scale is a validated tool used to measure neonatal outcomes, it relies on parent-report. Fourth, we assessed both female and male neonates together to preserve the statistical power of our analyses, however sex differences can influence the relationship between maternal mental health and motor outcome (Cao et al., 2014; Moss et al., 2017). Fifth, the use of antidepressants amongst the participants in our study was unknown and represents a potential confound. Finally, this was an online survey study and only participants with access to a computer and our participants are reflective of a higher socioeconomic status group, the BSMSS was used as a covariate to mitigate this limitation. 


\title{
Conclusion
}

As the present study demonstrates, adverse maternal mental health conditions during the COVID-19 pandemic not only affects mothers themselves but can also have negative downstream effects on developing infants. As the COVID-19 pandemic continues, it is important to develop an understanding of the many different ways the pandemic is negatively influencing mothers and their children so that we can find helpful and effective ways to intervene and support healthy long-term outcomes.

\section{Declarations}

\section{Data availability statement}

The datasets used and/or analysed during the current study are available from the corresponding author on reasonable request.

\section{Author Contributions:}

\begin{abstract}
A.P., E.S.N., Y.M., M.F.M., R.J.V.L., E.G.D., were involved in the study design, database variable creation, test material selection, and data acquisition design and execution of the data analytic strategy, reviewed and/or revised the final version of the manuscript.
\end{abstract}

A.P. and EGD contributed to the execution of the data analytic strategy, analyzed the data, prepared all figures and wrote the initial draft of the manuscript.

All authors approved the final manuscript as submitted and agree to be accountable for all aspects of the work.

\section{Acknowledgements}

The authors thank participants in this research. We thank Rachel Thorburn for her assistance with the administrative aspects of the study. The Canada First Research Excellence Fund (CFREF) through BrainsCAN provided the funding for this study.

\section{Tables}


Table 1. Characteristics of participants at time point 1 and time point 2 .

Time 1

Time 2

$n=117$

$\mathrm{n}=117$

Country, \% (No.)

Canada

USA

UK

India

Other

Unknown

Trimester, \% (No.)

$1^{\text {st }}$

$2^{\text {nd }}$

$3^{\text {rd }}$

30.8 (36)

$32.5(38)$

23.1 (27)

2.6 (3)

$10.2(12)$

0.85 (1)

0.9 (1)

$31.6(37)$

67.5 (79)

Education Level, \%, (No.)

Junior High/Middle School

0.9 (1)

High School

$6.0(7)$

University/College

$56.4(66)$

Graduate Degree

$35.9(42)$

Unknown

$0.9(1)$

Pandemic Month, \% (No.)

May - August 2020

March 2021

September - December 2020

January -

100 (117)

$0(0)$

$0(0)$

$32.6(38)$

$0(0)$

$67.6(79)$

Age, years, Median [IQR]

$31.0[29.0-$

34.75]

$53.0[43.0-$ 56.0] 20.0 [14.5 24.0]

$10.0[6.0-$ 14.0]
$31.0[29.0$ $-35.0]$

$53.0[48.0-$ 58.0] $15.0[9.8$ $-22.0]$ $7.0[4.0-$ 12.0]

\section{NEONATAL OUTCOMES}

Gestational Age, Weeks, Median [IQR]

Infant Birth Weight, Kgs, Median [IQR]

$40[38.9-$ $40.7]$

Motor Outcome Total Score, Median [IQR]

IQR, interquartile range; "Other" Countries: Austria, Chile, Germany, Greece, Hungary, Italy, Spain, Philippines, Portugal, Poland, Netherlands BSMSS; Barratt Simplified Measure of Social Status; PSS, perceived stress scale; EPDS, Edinburgh Postpartum Depression Scale.

Table 2. Four-cluster model participant demographics. 


\begin{tabular}{|c|c|c|c|c|c|}
\hline & $\begin{array}{c}\text { Cluster } 1 \\
n=17\end{array}$ & $\begin{array}{c}\text { Cluster } 2 \\
n=49\end{array}$ & $\begin{array}{c}\text { Cluster } 3 \\
n=27\end{array}$ & $\begin{array}{c}\text { Cluster } 4 \\
\mathrm{n}=14\end{array}$ & $\begin{array}{l}P \\
\text { value }\end{array}$ \\
\hline $\begin{array}{l}\text { EPDS Prenatal Score, } \\
\text { Median [IQR] }\end{array}$ & $\begin{array}{c}8.0 \\
{[6.0-13.0]}\end{array}$ & $\begin{array}{c}6.0 \\
{[3.0-9.0]}\end{array}$ & $\begin{array}{c}15.0 \\
{[12.0-} \\
18.0]\end{array}$ & $\begin{array}{l}12.5 \\
{[10.5-} \\
15.5]\end{array}$ & $<0.001$ \\
\hline $\begin{array}{c}\text { EPDS Postpartum } \\
\text { Score, } \\
\text { Median [IQR] }\end{array}$ & $\begin{array}{c}6.0 \\
{[3.0-7.0]}\end{array}$ & $\begin{array}{c}5.0 \\
{[3.0-7.0]}\end{array}$ & $\begin{array}{l}13.0 \\
{[11.0-}\end{array}$ & $\begin{array}{c}8.0 \\
{[5.0-16.5]}\end{array}$ & $<0.001$ \\
\hline $\begin{array}{c}\text { Motor Outcome, } \\
\text { Median [IQR] }\end{array}$ & $\begin{array}{c}6.0 \\
{[6.0-6.0]}\end{array}$ & $\begin{array}{c}6.0 \\
{[6.0-6.0]}\end{array}$ & 15.0] & 4.0 & $<0.001$ \\
\hline $\begin{array}{l}\text { Birth Weight (Kgs), } \\
\text { Median [IQR] }\end{array}$ & $\begin{array}{c}2.8 \\
{[2.5-3.05]}\end{array}$ & $\begin{array}{c}3.6 \\
{[3.4-3.9]}\end{array}$ & $\begin{array}{c}6.0 \\
{[6.0-6.0]}\end{array}$ & $\begin{array}{c}{[3.0-5.0]} \\
3.3\end{array}$ & $<0.001$ \\
\hline $\begin{array}{c}\text { Gestational Age (Days), } \\
\text { Median [IQR] }\end{array}$ & $\begin{array}{c}37.6 \\
{[35.71-} \\
38.9]\end{array}$ & $\begin{array}{c}40.3 \\
{[39.4-} \\
41.0]\end{array}$ & $\begin{array}{c}3.4 \\
{[3.2-3.6]} \\
40.1 \\
{[39.7-} \\
41.0]\end{array}$ & $\begin{array}{c}{[2.8-3.4]} \\
39.4 \\
{[38.2-} \\
40.1]\end{array}$ & $<0.001$ \\
\hline
\end{tabular}

IQR, interquartile range; EPDS, Edinburgh Postpartum Depression Scale. Cluster 1: lowaverage levels of pre- and post-natal depression, average Total Neonatal Motor Outcome, lower birth weight and lower gestational age at birth. Cluster 2: very low levels of pre- and post-natal depression, average Total Neonatal Motor Outcome, average birth weights and average gestational ages at birth. Cluster 3: high levels of both pre- and post-natal depression, average total motor outcome scores, average birth weight and average gestational age at birth. Cluster 4: high levels of both pre- and post-natal depression scores, impaired Total Neonatal Motor Outcome scores, low birth weight, and low-average gestational age at birth.

\section{References}

Abrams, S. M., Field, T., Scafidi, F., \& Prodromidis, M. (1995). Newborns of depressed mothers. Infant Mental Health Journal, 16(3), 233-239.

Adewuya A, Ola B, Aloba O. (2006) Anxiety disorders among Nigerian women in late pregnancy: A controlled study. Arch. Womens Ment. Health, 9, 325-328.

Ali, N. S., Mahmud, S., Khan, A., \& Ali, B. S. (2013). Impact of postpartum anxiety and depression on child's mental development from two peri-urban communities of Karachi, Pakistan: a quasiexperimental study. BMC psychiatry, 13(1), 1-12.

Barratt, W. (2006). The Barratt simplified measure of social status (BSMSS). Indiana State University.

Burger, M., Hoosain, M., Einspieler, C., Unger, M., \& Niehaus, D. (2020). Maternal perinatal mental health and infant and toddler neurodevelopment-Evidence from low and middle- income countries. A 
systematic review. Journal of Affective Disorders, 268, 158-172.

Cao, X., Laplante, D. P., Brunet, A., Ciampi, A., \& King, S. (2014). Prenatal maternal stress affects motor function in 51/2-year-old children: Project Ice Storm. Developmental

Psychobiology, 56(1), 117-125.

Chuang, C. H., Liao, H. F., Hsieh, W. S., Jeng, S. F., Su, Y. N., \& Chen, P. C. (2011). Maternal

psychosocial factors around delivery on development of 2-year-old children: A prospective cohort study. Journal of Paediatrics and Child Health, 47(1-2), 34-39.

Cohen, S., Kamarck, T., \& Mermelstein, R. (1983). A global measure of perceived stress. Journal of Health and Social Behavior, 385-396.

Cornish, A. M., McMahon, C. A., Ungerer, J. A., Barnett, B., Kowalenko, N., \& Tennant, C. (2005). Postpartum depression and infant cognitive and motor development in the second postpartum year: The impact of depression chronicity and infant gender. Infant Behavior and Development, 28(4), 407417.

Cox, J. L., Holden, J. M., \& Sagovsky, R. (1987). Detection of postpartum depression: development of the 10-item Edinburgh Postpartum Depression Scale. The British Journal of Psychiatry, 150(6), 782786.

Diego, M. A., Field, T., \& Hernandez-Reif, M. (2005). Prepartum, postpartum and chronic depression effects on neonatal behavior. Infant Behavior and Development, 28(2), 155- 164.

Felice E, Saliba J, Grech V, Cox J. (2006) Validation of the Maltese version of the Edinburgh Postnatal Depression Scale. Arch. Womens Ment. Health, 9, 75-80.

Field, T., Diego, M., Dieter, J., Hernandez-Reif, M., Schanberg, S., Kuhn, C., ... \& Bendell, D. $\quad$ (2004). Prenatal depression effects on the fetus and the newborn. Infant Behavior and Development, 27(2), 216229.

Koutra, K., Chatzi, L., Bagkeris, M., Vassilaki, M., Bitsios, P., \& Kogevinas, M. (2013). Antenatal and postnatal maternal mental health as determinants of infant neurodevelopment at 18 months of age in a mother-child cohort (Rhea Study) in Crete, Greece. Social Psychiatry and Psychiatric Epidemiology, 48(8), 1335-1345.

Lebel, C., MacKinnon, A., Bagshawe, M., Tomfohr-Madsen, L., \& Giesbrecht, G. (2020). Elevated depression and anxiety symptoms among pregnant individuals during the COVID-19 pandemic. Journal of Affective Disorders, 277, 5-13.

Mitchell AM, Crane PA, Kim Y (2008) Perceived stress in survivors of suicide: psychometric properties of the Perceived Stress Scale. Res Nurs Health, 31(6):576-85. 
Moss, K. M., Simcock, G., Cobham, V., Kildea, S., Elgbeili, G., Laplante, D. P., \& King, S.

(2017). A potential psychological mechanism linking disaster-related prenatal maternal stress with child cognitive and motor development at 16 months: The QF2011 Queensland Flood Study. Developmental Psychology, 53(4), 629.

Murray D, Cox JL. (1990) Screening for depression during pregnancy with the Edinburgh Postpartum Depression Scale (EPDS). J. Reprod. Infant Psychol. 899-107.

Nasreen, H. E., Kabir, Z. N., Forsell, Y., \& Edhborg, M. (2013). Impact of maternal depressive symptoms and infant temperament on early infant growth and motor development: results from a population based study in Bangladesh. Journal of affective disorders, 146(2), 254- 261.

O'Leary, N., Jairaj, C., Molloy, E. J., McAuliffe, F. M., Nixon, E., \& O'Keane, V. (2019). Antenatal depression and the impact on infant cognitive, language and motor development at six and twelve months postpartum. Early Human Development, 134, 4146.

Oyetunji, A., \& Chandra, P. (2020). Postpartum stress and infant outcome: A review of current literature. Psychiatry research, 284, 112769.

Papadopoulos, A., Nichols, E. S., Mohsenzadeh, Y., Giroux, I., Mottola, M. F., Van Lieshout, R. J., \& Duerden, E. G. (2021). Depression in pregnant women with and without COVID- 19. BJPsych Open, $7(5)$.

Pinheiro, K. A. T., Pinheiro, R. T., Coelho, F. M. D. C., da Silva, R. A., Quevedo, L. A., Schwanz, C. C., ... \& Oses, J. P. (2014). Serum NGF, BDNF and IL-6 levels in postpartum mothers as predictors of infant development: the influence of affective disorders. PLoS One, 9(4), e94581.

Racine, N., Madigan, S., Plamondon, A., Hetherington, E., McDonald, S., \& Tough, S. (2018). Maternal psychosocial risk profiles in pregnancy: Associations with postpartum maternal health and child outcomes. Clinical Psychological Science, 6(6), 783-796.

Simcock, G., Kildea, S., Elgbeili, G., Laplante, D. P., Stapleton, H., Cobham, V., \& King, S. (2016). Agerelated changes in the effects of stress in pregnancy on infant motor development by maternal report: The Queensland Flood Study. Developmental Psychobiology, 58(5), 640-659.

Sacchi, C., De Carli, P., Vieno, A., Piallini, G., Zoia, S., \& Simonelli, A. (2018). Does infant negative emotionality moderate the effect of maternal depression on motor development?. Early human development, 119, 56-61.

Slomian, J., Honvo, G., Emonts, P., Reginster, J. Y., \& Bruyère, O. (2019). Consequences of maternal postpartum depression: A systematic review of maternal and infant outcomes. Women's Health, 15, 1745506519844044. 
Stepowicz, A., Wencka, B., Bieńkiewicz, J., Horzelski, W., \& Grzesiak, M. (2020). Stress and Anxiety Levels in Pregnant and Post-Partum Women during the COVID-19 Pandemic. International Journal of Environmental Research and Public Health, 17(24), 9450.

Stewart SL, lantosca JM, Klassen J, Tucker M, Fisman S, McClean J. (2017) interRAl 0-3 Assessment Form and User's Manual. Version 1. Washington, DC: interRAl publications.

Suárez-Rico, B. V., Estrada-Gutierrez, G., Sánchez-Martínez, M., Perichart-Perera, O., RodríguezHernández, C., González-Leyva, C., ... \& Reyes-Muñoz, E. (2021). Prevalence of depression, anxiety, and perceived stress in postpartum Mexican women during the COVID-19 lockdown. International Journal of Environmental Research and Public Health, 18(9), 4627.

Wu, Y., Zhang, C., Liu, H., Duan, C., Li, C., Fan, J., ... \& Huang, H. F. (2020). Perinatal depressive and anxiety symptoms of pregnant women during the coronavirus disease 2019 outbreak in China. American Journal of Obstetrics and Gynecology, 223(2), 240- e1.

Zanardo, V., Manghina, V., Giliberti, L., Vettore, M., Severino, L., \& Straface, G. (2020). Psychological impact of COVID-19 quarantine measures in northeastern Italy on mothers in the immediate postpartum period. International Journal of Gynecology \& $\quad$ Obstetrics, 150(2), 184-188.

Zilver, S. J. M., Broekman, B. F. P., Hendrix, Y. M. G. A., de Leeuw, R. A., Mentzel, S. V., van Pampus, M. G., \& de Groot, C. J. M. (2021). Stress, anxiety and depression in 1466 pregnant women during and before the COVID-19 pandemic: a Dutch cohort study. Journal of Psychosomatic Obstetrics \& Gynecology, 42(2), 108-114.

\section{Figures}




\section{5}

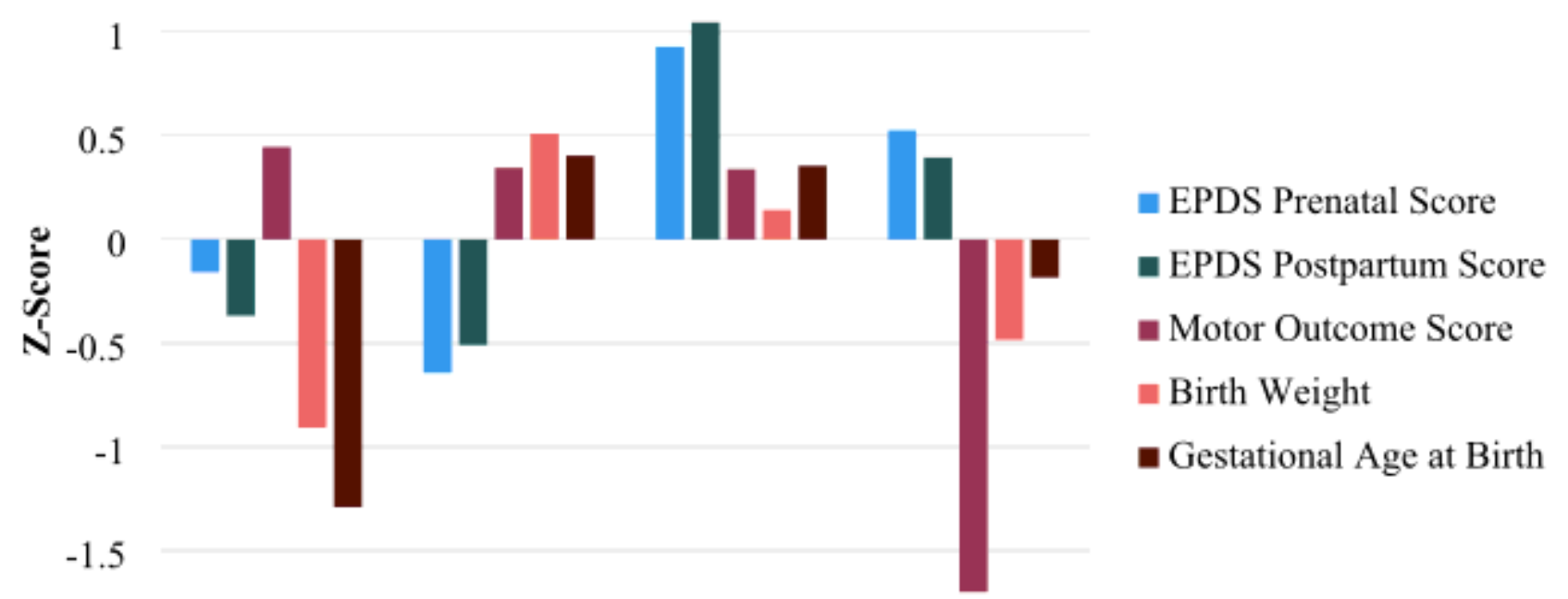

$-2$

\section{$\begin{array}{llll}\text { Cluster } 1 & \text { Cluster } 2 & \text { Cluster } 3 & \text { Cluster } 4\end{array}$}

\section{Figure 1}

K-means clustering based on pre- and post-natal depression, Total Neonatal Motor Outcome, birth weight, and gestational age at birth.

The four-cluster model is depicted above. Cluster 1 is characterized by low-average levels of pre-and post-natal depression, average Total Neonatal Motor Outcome, a low birth weight and a low gestational age at birth. Cluster 2 is characterized by very low levels of pre- and post-natal depression, average Total Neonatal Motor Outcome, average birth weights and average gestational ages at birth. Cluster 3 is characterized by high levels of both pre- and post-natal depression, average Total Neonatal Motor Outcome scores, average birth weight and average gestational age at birth. Cluster 4 is characterized by high levels of both pre- and post- natal depression scores, impaired Total Neonatal Motor Outcome scores, low birth weight, and low-average gestational age at birth. 